\title{
A Mapping Study about Data Lakes: An Improved Definition and Possible Architectures
}

\author{
Julia Couto, Olimar Borges, Duncan Ruiz, Sabrina Marczak, and Rafael Prikladnicki \\ School of Technology, PUCRS - Pontifical Catholic University of Rio Grande do Sul - Porto Alegre, Brazil \\ \{julia.couto, olimar.borges\}@edu.pucrs.br, \{duncan.ruiz, sabrina.marczak, rafaelp\}@ pucrs.br
}

\begin{abstract}
In the past few years, data lakes emerged as a trending topic in big data technologies. Although literature presents different points of view related to its functionalities, it serves mainly to store a variety of data in a big data context. In this paper, we aim to identify and analyze data lake definitions and possible architectures. Our methodology was composed of a systematic literature mapping based on PRISMA, software engineering best practices to perform reviews, and Kappa method to assess results' quality. We performed the search in eight different electronic databases to achieve a wide variety of publishers in Computer Science. We first identified 662 papers matching our search criteria; after filtering, we selected 87 papers for review. We found that the term data lakes was first defined by James Dixon in 2010. We also found that the term is often related to raw data repositories. From the identified definitions, we propose a new one as a means to better state what data lakes refer to and improve how the community use them. Moreover, we foind that Hadoop and its ecosystem compose the most used toolset to create data lakes, revealing that this is the mainstream in architectures for data lakes as of today's available technologies.
\end{abstract}

Index Terms-Data lakes, Big Data, Literature review, PRISMA, Hadoop.

\section{INTRODUCTION}

Data lakes are a recent and trending topic in big data context [24], [81]. It is often referred as an architecture to store big data. They are often compared to traditional data warehouses, but both concepts differ in several aspects. For instance, unlike data warehouses, data lakes can easily scale and have the ability to store schema-less and multivariate data that will be processed just when information needs to be extracted from the stored dataset [8], [13], [38], [44], [80]. This native characteristic makes data lakes quite suitable for big data ecosystems.

Although the concept was first used in earlier 2010 [20], it was adopted by academia only a couple of years later. Thus, there is no consolidated and universally accepted definition, and its functionalities vary according to the context. For example, some say it is only a data repository [30], [43], [86], while others say it is a complete ecosystem, from data acquisition to information visualization [9], [55], [62]. By having these different functionalities, data lakes also present different possible architecture configurations.

The foundation architecture to create a data lake may be different according to some variables, such as its purpose, the skills of the people responsible for creating it, the available

DOI reference number: 10.18293/SEKE2019-129 infrastructure, and tools. For example, when considering the Hadoop Ecosystem, there are more than a hundred tools available for it [22], with thousands of possible combinations among their use. Knowing which ones are most commonly used or those that are most commonly reported in the literature may be useful for novices on big data, who need to set up an initial data lake, and also for those who are more experienced users, who might want to know new tools to add advanced features to an existing data lake.

Therefore, the aim of our literature review was to better understand what definitions have been used by the research community for the term 'data lake', and to propose a more comprehensive definition to facilitate and improve its use. Additionally, we also aimed to identify which big data architectures are used to build a data lake as well as to map the associated tools to do so.

More specifically, we performed a systematic mapping study in eight electronic databases. From the 662 identified papers, we selected 87 papers for review after filtering. We used the PRISMA checklist [59] to help us improve the quality report of our study, and the process suggested by Kitchenhan et al. [11] to plan the steps to be followed. To enhance results quality and measure the level of agreement between the researchers, we used the Kappa [54] method. To reduce bias, two researchers analyzed the selected papers and two others were consulted to resolve disagreements.

Our study revealed that James Dixon was the first author to use the concept of data lake to refer to a solution to store raw data in a Hadoop ecosystem, in 2010 [20]. The first conference paper to cite the term is from 2014, by O'Leary [63]. We also found that the terms most frequently associated to data lake are: store, raw, repository, formats, analysis, storage, processed, and sources. About data lake architectures, Hadoop is the most commonly used, stand-alone or in combination with other tools, such as Spark and NoSQL databases. The remaining sections present our study and results in details.

\section{MATERIALS AND METHODS}

A systematic mapping study, also known as mapping study (MS), is a type of literature review, a research method largely used to understand the state of art of some subject, and it allows us to map its origins and also how it developed over time, based on research questions. To develop our MS, we follow the process defined by Brereton et al [11]. These authors suggest three phases, namely Plan, Conduct, and Document 
TABLE I

PICO AND PICO DEFINITIONS

\begin{tabular}{|c|c|}
\hline PICO & PICo \\
\hline Population: Big data systems & $\begin{array}{l}\text { Population: Big data sys- } \\
\text { tems }\end{array}$ \\
\hline $\begin{array}{l}\text { Intervention: Data lakes } \\
\text { Comparison: Definition of data lakes }\end{array}$ & $\begin{array}{l}\text { Interest: Definitions and } \\
\text { architectures }\end{array}$ \\
\hline $\begin{array}{l}\text { Outcome: Definition of data lakes and big } \\
\text { data architectures in data lakes ecosystems }\end{array}$ & Context: Data lakes \\
\hline
\end{tabular}

the review, having ten stages to develop these phases. We also use the Preferred Reporting Items for Systematic Review and Meta-Analysis Protocols (PRISMA-P) [59] checklist, that has a set of items that must be addressed to report a systematic review, as described next.

\section{A. Plan Review}

In the Planning Phase, we defined research questions, and developed and assessed the review protocol. This phase must be done carefully because of it basis all subsequent research.

1) Specify Research Question: Our main objective is to answer the following Research Question (RQ): What are the definitions and possible big data architectures in data lake ecosystems? To better explore the papers, we splitted RQ into two, so each accepted paper can answer one or two questions: 1) What are the most common definitions to the term data lake? 2) Which system architectures are reported to be used in data lake ecosystems?

Aiming to limit and clarify our scope, we followed the PICO (Population, Intervention, Comparison, and Outcome) and PICo (Population, Interest, and Context) methods. These were initially developed by Sacket [72], to facilitate the elaboration of research definitions. PICO are most used for quantitative studies, while qualitative studies usually apply PICo [89]. As a MS can contain both qualitative and quantitative data, we used PICO and PICo to help us elaborate our research question. We present the scope of our research in Table I]

2) Develop Review Protocol: We developed and applied our search protocol using digital libraries available in the internet. We defined control studies so we could validate our search strings. A control study is an primary study resulting from systematized research, and which is known to answer our research questions. We used it to check if the search strings are adequate: if the control papers are not returned during string adjustments, the strings need to be adjusted until they do so. We used the two papers listed in Table II as control papers. Table III lists the used eletronic databases and search strings.

TABLE II

CONTROL PAPERS

\begin{tabular}{l|l}
\hline $\begin{array}{l}\text { Control } \\
\text { Study 1 }\end{array}$ & $\begin{array}{l}\text { Terrizzano, Ignacio G., et al. "Data Wrangling: The Challeng- } \\
\text { ing Journey from the Wild to the Lake." CIDR. 2015. [87| }\end{array}$ \\
\hline $\begin{array}{l}\text { Control } \\
\text { Study 2 }\end{array}$ & $\begin{array}{l}\text { Madera, Cedrine, and Anne Laurent. "The next information } \\
\text { architecture evolution: the data lake wave." Proc. Int'l Conf. } \\
\text { on Management of Digital EcoSystems. ACM, 2016. [50| }\end{array}$ \\
\hline
\end{tabular}

TABLE III

SEARCH STRINGS FOR EACH ELECTRONIC DATABASES.

\begin{tabular}{|c|c|}
\hline Database & Search String \\
\hline Springer & $\begin{array}{l}\text { search?query="data+lake"\&facet- } \\
\text { language="En"\&date-facet- } \\
\text { mode=between\&showAll=true\&facet- } \\
\text { discipline="Computer+Science" }\end{array}$ \\
\hline Google Scholar & allintitle: "data lake" \\
\hline $\begin{array}{l}\text { Scopus } \\
\end{array}$ & $\begin{array}{l}\text { TITLE-ABS-KEY ( data lake ) AND ( LIMIT-TO ( } \\
\text { SUBJAREA, "COMP" ) }\end{array}$ \\
\hline Web of Science & $\begin{array}{l}\text { (from all databases): TOPIC: ("data lake") OR TI- } \\
\text { TLE: ("data lake") OR AUTHOR IDENTIFIER: } \\
\text { ("data lake") }\end{array}$ \\
\hline IEEE Xplore & $\begin{array}{l}\text { (("Document Title":"data lake") OR "Ab- } \\
\text { stract":"data lake") OR "Author Keywords":"data } \\
\text { lake") }\end{array}$ \\
\hline $\begin{array}{l}\text { Science Direct } \\
\text { arXiv }\end{array}$ & $\begin{array}{l}\text { Title, abstract, keywords: "data lake" } \\
\text { order: -announced_date_first; page_size: 50; pri- } \\
\text { mary_classification: cs; terms: AND all="data lake" }\end{array}$ \\
\hline $\mathrm{ACM}$ & $\begin{array}{l}\text { acmdlTitle:(+data +lake) AND recordAbstract:(+data } \\
\text { +lake) AND keywords.author.keyword:(+data +lake) }\end{array}$ \\
\hline
\end{tabular}

It is important to note that we did not set a data range for the search. Returned results from as early as 1969 to 2013 referring to data lakes, upon inspection, were identified to discuss geological lakes. Thus, given that these do not relate to Computer Science, we discarded them; having the first paper of interest reported in 2014.

3) Validate Review Protocol: Two researchers developed the review protocol, who made several trials changing the search string to obtain results relevant and aligned to the research question. Then, the protocol was validated by two other senior researchers with a $\mathrm{PhD}$ degree in Computer Science. One of these researchers is a domain specialist in databases and big data and the other in research methodology. The study was conducted based on the updated protocol upon their reviews, as presented next.

\section{B. Conduct Review}

We conducted the study as per the defined protocol.

1) Identify Relevant Research: We applied the defined search string and, from the results, generated a bibtex file format for each electronic database. Bibtex is a plain-text fileformat that contains lists of references, with information about all paper that matches our search criteria.

2) Select Primary Studies: To reduce bias, we splitted the papers to be analyzed between two researchers. We start selection phase with 1 st researcher reviewing and marking each paper as accepted or rejected. Then, we perform three review rounds, based on Kappa method [54], each one containing a random sample of $5 \%$ of the papers population that was reviewed by the second researcher. We used the Kappa statistic [54] to measure the level of agreement between the researchers. Kappa result is based on the number of answers with the same result for both observers [46]. Its maximum value is 1 , when the researchers have almost perfect agreement, and it tends to zero or less when there are no agreement between them. 
TABLE IV

KAPPA RESULTS, BASED ON LANDIS \& KOCH [46].

\begin{tabular}{llccc}
\hline Kappa values & Agreement & $1^{\circ}$ round & $2^{\circ}$ round & $3^{\circ}$ round \\
\hline$<0$ & Poor & & & \\
$0-0.20$ & Slight & & & \\
$0,21-0,40$ & Fair & \multirow{2}{*}{0.42} & & \\
$0,41-0,60$ & Moderate & & 0.64 & \\
$0,61-0,80$ & Substantial & & & 0.82 \\
$0,81-1$ & Almost perfect & & \\
\hline
\end{tabular}

For each round, the 2nd researcher received a sample, analyzed each paper, and marked each one as accepted or rejected. Then, we compared the answers: if 1st and 2nd researchers accepted the same paper, we have an agreement in that paper. Then, we calculate Kappa value for the round. After that, in the papers where there is no agreement, the two main researchers discuss about the paper to reach a consensus. If there is still no consensus, the other researchers are contacted to help decide.

Landis \& Koch [46] define a scale to interpret the Kappa results (see Table IV). We can also see in this table the results from the 3 rounds of analysis. We can see that the level of agreement increased, from moderate in the 1st round to substantial in the 2nd one, and in the last we achieved almost perfect agreement. In each iteration, we discussed the results and the reasons why some papers had been selected and other had not, improving the agreement between the researchers on the next iteration. From that, the second researcher received the second-half of papers to independently review.

3) Assess Study Quality: In order to retrieve interesting results related to the research topic, we defined inclusion and exclusion criteria for the papers. To be accepted, papers must meet all the following criteria: 1) $\mathrm{Be}$ a qualitative or quantitative research on data lakes in data management; 2) Present a complete study in electronic format; 3) Be a conference paper, review or journal. On the other hand, papers we rejected meet at least one of the following criteria: 1) Incomplete or short paper (less than 3 pages); 2) Unavailable for download; 3) Not about data lakes in data management; 4) Duplicated study; 5) Written in another language than English; 6) Conference proceedings index.

4) Extract Required Data: To help us organize and classify the papers, we used a tool named StArt (State of the Art through Systematic Review $\sqrt{1}$ StArt was developed by the Federal University of São Carlos, Brazil, and it helps researchers in the process of systematic literature reviews. StArt has a execution phase with 3 processes: studies identification, selection, and extraction. We first register the protocol, and then we register each database and import its bib file, then use StArt to help keep record of selected papers. After finishing all data extraction using StArt, we exported the results to a Google Sheets, so we could analyze the data.

5) Synthesize Data: We used Google Sheets to help analyze and summarize our results. It also helped to work collabora-

\footnotetext{
${ }^{1}$ Available at: http://lapes.dc.ufscar.br/tools/start_tool
}

TABLE V

PAPERS PER ELECTRONIC DATABASES.

\begin{tabular}{|c|c|c|}
\hline Source & Initial & Accepted \\
\hline Scopus & 108 & 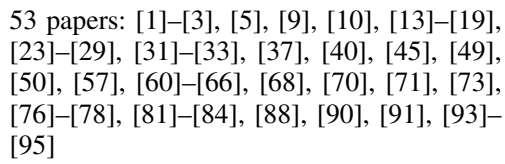 \\
\hline Springer & 222 & $\begin{array}{l}20 \text { papers: [4], [6], [12], [21], [30], [36], } \\
{[38],[39],[41]-[43],[47],[51], \mid[53],[69],} \\
{[74],[79],[85],[86],[92]}\end{array}$ \\
\hline Google Scholar & 197 & 6 papers: [8], |34], [56], |67], [80], |87] \\
\hline Web of Science & 71 & 4 papers: [7], [44], |48], |58| \\
\hline Science Direct & 19 & 2 papers: $[35], 775]$ \\
\hline IEEE Xplore & 32 & 1 paper: 52 \\
\hline $\operatorname{arXiv}$ & 7 & 1 paper: $[55$ \\
\hline$\overline{\mathrm{ACM}}$ & 6 & 0 papers \\
\hline
\end{tabular}

tively, as Google Sheets is available online.

\section{Document Review}

1) Write Review Report: After finishing answering the questions of the MS, we use our protocol as a basis to document the Review. Results are presented in Section III.

2) Validate Report: Once we finished the report, it was independently reviewed by three senior researchers. Each one read and suggest improvements that were adjusted to this final version.

\section{RESUlTS}

We started with 662 papers retrieved from the initial search through the web engines. During the process, we identified that 155 are duplicated, and 419 were rejected according to exclusion criteria previously explained. At the end of MS process, we accepted 87 papers, published between 2014 and 2018. Table V presents the distribution of papers per database. In this table, we can see that most of the papers came from Springer and Google Scholar. It happens because Springer does not allow us to refine the filter of the studies, so results contains lots of books and books chapters, which we reject, as we explained in inclusion and exclusion criteria. Google Scholar, in the same way, does not allow complementary filters, frequently redirects to other engines, and it also brings a lot of websites and non-scientific reports among the results.

Among the rejected papers, 75 were published before 2010, when the term data lake was first used in big data context. We have to manually reject the older ones due to the fact that in most electronic databases we cannot filter results to show only Computer Science related studies. The papers previous to 2010 are mostly from Geology or Civil Engineering. We found that there is an increasing interest in data lakes, since 2014, with most papers being published in 2018.

Other interesting aspect we can see in Table $\mathrm{V}$ is that more than half papers we accepted are from Scopus. It happens due to the fact that Scopus is the largest database of abstracts and scientific citations, compiling more than 71 million records, 23 million titles and 5,000 publishers, among them ACM, Elsevier, IEEE, Springer, etc. So, we probably accepted papers 
from other databases using Scopus reference, and then it was marked as duplicated in the original database version.

\section{A. What are the most common definitions to the term data} lake?

From the 87 papers we accepted, 71 present data lake definitions. We read each one and copied the definition they present to the term data lake. Then, we created a unique text containing all definitions, and we passed through a web tool to count the words. This tool removed the stopwords, the most frequent terms in English, that are usually removed before natural language processing. Then, it returned a list containing all the other words and the amount of times they appear in the text. We analyzed the resulting list and grouped the variances in the same word, by the most frequent one: e.g.: analyses, analyzing, and analysis were grouped into analysis. Table VI presents the top 30 most frequent words. Then, based on the most frequent words, we create a word cloud (Figure 1) and a new definition to the term data lake, presented below.

Data lake is a central repository system for storage, processing, and analysis of raw data, in which the data is kept in its original format and is processed to be queried only when needed. It can store a varied amount of formats in big data ecosystems, from unstructured, semi-structured, to structured data sources.

During our analysis, we mapped who the authors of the papers references when using a definition for data lakes. We found that James Dixon was the first one to use the term lake in big data context, in a post in its blog in 2010 [20], and he is referenced by ten papers [4], [6], [17], [32], [38], [44], [62], [63], [67], [91]. The first author to reference Dixon's Concept in academic context was O'Leary [63], in a paper published in 2014. We also discovered the most cited academic definition for data lakes is from Terrizzano et al. [87], mentioned in twelve papers [5], [26], [27], [33], [34], [52], [66], [78], [82], [84], [91], [93].

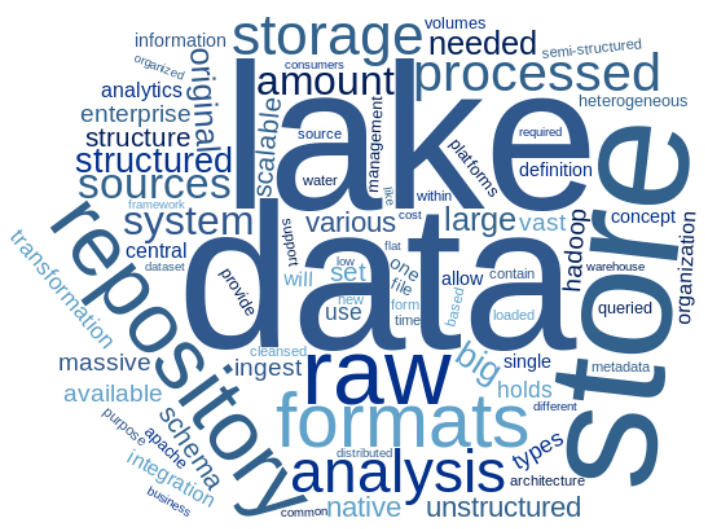

Fig. 1. Word cloud with the terms most commonly related to data lakes
TABLE VI

30 MOST FREQUENT WORDS RELATED TO DATA LAKE DEFINITIONS.

\begin{tabular}{rl|rl|rl}
\hline Word & Count & Word & Count & Word & Count \\
\hline data & 357 & amount & 20 & scalable & 13 \\
lake & 105 & system & 20 & schema & 13 \\
store & 81 & big & 17 & set & 13 \\
raw & 45 & structured & 17 & structure & 13 \\
repository & 42 & large & 16 & available & 12 \\
formats & 37 & needed & 16 & enterprise & 12 \\
analysis & 29 & original & 16 & Hadoop & 12 \\
storage & 28 & native & 14 & ingest & 12 \\
processed & 26 & unstructured & 14 & massive & 12 \\
sources & 21 & various & 14 & vast & 12 \\
\hline
\end{tabular}

\section{B. Which system architectures are reported to be used in data} lakes ecosystems?

System architectures are formed by a set of tools that work together to achieve the environment objective. Among the 87 papers we mapped, we identified 117 different tools used in data lake architectures. Table VII lists the tools cited more than once. Of all the tools, Apache Hadoop was the most mentioned, having 37 citations ( [1], [6], [8], [9], [12], [18], [23]-[25], [27], [28], [30], [31], [40], [42]-[44], [47], [48], [50], [52], [55], [61], [65], [67]-[71], [73], [76], [80], [82][86], [88]). Hadoop is a platform that allows distributed processing of large data sets in computer clusters. It is currently considered a complete ecosystem to Big Data storage and processing. We list and categorize the remaining tools in five groups, described as follows.

1) Ingestion: Class of tools that work on data acquisition and collection, from the most varied sources. In this group, the most cited tool is Apache Kafka, which consists of a highcapacity, low latency distributed streaming platform for realtime data processing.

2) Storage: Represented by tools to store, integrate and normalize data. Besides Hadoop, Apache Cassandra and MongoDB, are the most popular for this group. Apache Cassandra is a highly scalable, column-driven distributed database that has a data model based on Google's BigTable. MongoDB is a multi-platform, document-oriented database that stores data in JSON documents with the dynamic schema. It can be considered the most famous NoSQL database on the market.

3) Processing: Tools in this group are responsible for analyzing, processing and transforming the raw data, so we can extract information from it. In this group, Apache Spark is the most cited in all papers, besides Apache Hadoop. It is a framework for distributed computing that provides an interface for clustered programming with parallelism and fault tolerance.

4) Presentation: Tools that help us make sense of the data, in a visual manner. In this case, Microsoft Power BI and Tableau are the most mentioned in the papers. Power BI is a cloud-based Business Analytics service focused on presenting information across dashboards. Tableau is a software for interactive data visualization. 
TABLE VII

ARCHITECTURES: THE MOST USED TOOLS IN DATA LAKES

\begin{tabular}{|c|c|c|}
\hline Tool & Amount & Papers \\
\hline \multicolumn{3}{|l|}{ 1) Ingestion } \\
\hline Apache Kafka & 10 & 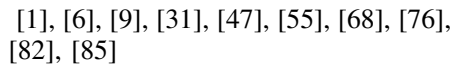 \\
\hline Apache Flume & 7 & |T], |6], [27], [52], [61], |70], [83] \\
\hline Apache Sqoop & 5 & [1], [27], โ47], |52], |55] \\
\hline Apache Nin & 3 & {$[\overline{1}|,| \overline{55}|,| \overline{76} \mid$} \\
\hline Komadu & 2 & [83], [84] \\
\hline Talend Studio & 2 & $\mid 1],[93]$ \\
\hline \multicolumn{3}{|l|}{ 2) Storage } \\
\hline Apache Cassandra & 6 & [1], |6], \21], [40], |41], \45] \\
\hline MongoDB & 6 & [16], $\mid 33], \mid 35], \mid 41], \mid 43], \mid 62]$ \\
\hline Apache HBase & 4 & {$[1,[31],[47, \sqrt[69]{ }$} \\
\hline MySQL & 4 & {$[\overline{1}|,| \overline{33}], \overline{43}|,| \overline{84} \mid$} \\
\hline Neo4J & 3 & {$[\overline{65}|,| \overline{85}],|\overline{91}|$} \\
\hline Oracle & 3 & $\mid 1], \mid 12],|42|$ \\
\hline Apache Mahout & 2 & $[1], \mid 42]$ \\
\hline GlusterFS & 2 & [48], [64] \\
\hline PostgreSQL & 2 & [41], [78] \\
\hline \multicolumn{3}{|l|}{ 3) Processing } \\
\hline Apache Spark & 26 & 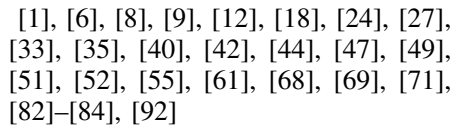 \\
\hline Apache Hive & 11 & $\frac{\mid 11],[6],[9],[12]}{[55],}, \frac{[25]}{\lfloor 61],[69]},[27],[31]$, [43], \\
\hline Apache Storm & 7 & |1], |42], |55|, |65], |82]--|84] \\
\hline Apache Impala & 4 & {$[\overline{12}|| 61],,|69|,|88|$} \\
\hline Apache Drill & 4 & $|\overline{12}|,|\overline{43}|,|\overline{65}|,|\overline{71}|$ \\
\hline Apache Oozie & 4 & $[\pi], \mid 27],[52],[55]$ \\
\hline Python & 4 & [1], |16], |35], |88| \\
\hline Apache Flink & 3 & $|6|,|44|,|82|$ \\
\hline Apache Pig & 3 & [1], |27], |52] \\
\hline Apache POI & 2 & {$[1],|66|$} \\
\hline Kepler & 2 & [83], [84] \\
\hline Shiny & 2 & {$[\overline{27}],[\overline{52}]$} \\
\hline Splunk & 2 & $|\overline{11}| 69 \mid$, \\
\hline WEKA & 2 & [42], [93] \\
\hline \multicolumn{3}{|l|}{ 4) Presentantion } \\
\hline Microsoft Power BI & 2 & [88], [90] \\
\hline Tableau & 2 & $[1], \mid 61]$ \\
\hline \multicolumn{3}{|l|}{ 5) Security } \\
\hline Apache Ranger & 4 & [31], |55], |70], |71] \\
\hline Kerberos & 3 & $|\overline{55}|,|\overline{70}|,|\overline{\mid 71}| \square$ \\
\hline Apache Ambari & 2 & {$[\overline{55}|,| \overline{71} \mid$} \\
\hline Apache Knox & 2 & [31], [71] \\
\hline Apache Sentry & 2 & {$[31],[71]$} \\
\hline
\end{tabular}

5) Security: Includes tools to manage system authentication and authorization, assure data security, permit auditing, and allow data encryption. Apache Ranger is the most mentioned. It is a framework for activating, monitoring and managing data security in the Apache Hadoop ecosystem. According to our analysis, the Apache Software Foundation (ASF) develops most of the tools reported in the studies for data lake architectures, helping creating the most used ecosystems.

\section{CONCLUSION}

In this paper, we presented a systematic mapping study to better explain data lakes definition and architecture. We started with 662 papers, and we end up with 87 in the final set, after our criteria selection. The papers we selected are from 2014 to 2018 , and came from eight different electronic databases.
We learned that the term data lake was first used in 2010 to designate a big data system. We proposed a new definition from the selected papers in our study for the concept data lake. We also found that Hadoop and its ecosystem comprises the most frequent architecture to built data lakes.

One limitation of our study is that we choose to limit the search only to the papers that have the term "data lake". We know that many researchers can be working with data lakes without using this buzzword, but as we want to know its definition, we chose to accept that limitation. For future work, we plan to further the investigation on the used tools and architectures, discussing the categories we listed and building a framework to help beginners to choose the best configuration according to its needs.

\section{REFERENCES}

[1] F. Ahmad et al., "Qos lake: Challenges, design and technologies," in SigTelCom, 2017, pp. 65-70.

[2] A. Ahmadov et al., "Towards a hybrid imputation approach using web tables," in BDC. IEEE/ACM, 2015, pp. 21-30.

[3] H. Alili et al., "Quality based data integration for enriching user data sources in service lakes," in ICWS. IEEE, 2018, pp. 163-170.

[4] H. Alrehamy et al., "Semlinker: automating big data integration for casual users," Journal of Big Data, vol. 5, no. 1, p. 14, 2018.

[5] A. Alserafi et al., "Towards information profiling: Data lake content metadata management," in ICDMW. IEEE, 2016, pp. 178-185.

[6] S. Auer et al., "The bigdataeurope platform - supporting the variety dimension of big data," in ICWE. Springer, 2017, pp. 41-59.

[7] A. Beheshti et al., "Coredb: A data lake service," in CIKM. ACM, 2017, pp. 2451-2454.

[8] R. Benaissa et al., "Clustering approach for data lake based on medoid's ranking strategy," in CSA. Springer, 2018, pp. 250-260.

[9] M. Bhandarkar, "Adbench: A complete benchmark for modern data pipelines," in TPCTC. Springer, 2017, pp. 107-120.

[10] W. Brackenbury et al., "Draining the data swamp: A similarity-based approach," in HILDA. ACM, 2018, pp. 13:1-13:7.

[11] P. Brereton et al., "Lessons from applying the systematic literature review process within the software engineering domain," Journal of systems and software, vol. 80, no. 4, pp. 571-583, 2007.

[12] P. Ceravolo et al., "Big data semantics," Journal on Data Semantics, vol. 7, no. 2, pp. 65-85, 2018.

[13] B. Cha et al., "International network performance and security testing based on dist. abyss storage cluster and draft of data lake framework," Security and Communication Networks, vol. 2018, pp. 1-14, 2018.

[14] H. Chen et al., "An early functional and performance experiment of the marfs hybrid storage ecosystem," in IC2E. IEEE, 2017, pp. 59-66.

[15] Y. Chen et al., "Enhancing the data privacy for public data lakes," in ICASI. IEEE, 2018, pp. 1065-1068.

[16] A. Ciociola et al., "Umap: Urban mobility analysis platform to harvest car sharing data," in SmartWorld. IEEE, 2017, pp. 1-8.

[17] N. Dessì et al., "Increasing open government data transparency with spatial dimension," in WETICE. IEEE, 2016, pp. 247-249.

[18] A. Dholakia et al., "Designing a high performance cluster for large-scale sql-on-hadoop analytics," in Big Data. IEEE, 2017, pp. 1701-1703.

[19] C. Diamantini et al., "An approach to extracting thematic views from highly heterogeneous sources of a data lake," in SEBD, 2018, pp. 1-12.

[20] J. Dixon, "Pentaho, hadoop, and data lakes," https://jamesdixon. wordpress.com/2010/10/14/ 2010, accessed: 2019-02-20.

[21] H. Dutta, "Graph based data governance model for real time data ingestion," CSI Trans. on ICT, vol. 3, no. 2, pp. 119-125, 2015.

[22] J. R. et al., "The hadoop ecosystem table," https://hadoopecosystemtable. github.io/ 2019, accessed: 2019-02-22.

[23] H. Fang, "Managing data lakes in big data era," in CYBER. IEEE, 2015, pp. 820-824.

[24] M. Farid et al., "Clams: Bringing quality to data lakes," in ICMD. ACM, 2016, pp. 2089-2092.

[25] A. Farrugia et al., "Towards social network analytics for understanding and managing enterprise data lakes," in ASONAM. IEEE/ACM, 2016, pp. 1213-1220. 
[26] Y. Gao et al., "Navigating the data lake with datamaran," CoRR, vol. abs/1708.08905, 2017.

[27] I. García et al., "Towards a scalable architecture for flight data management," in DATA, INSTICC. SciTePress, 2017, pp. 263-268.

[28] S. Gollapudi, "Aggregating financial services data without assumptions," in ICSC. IEEE, 2015, pp. 312-315.

[29] N. Golov et al., "Big data normalization for massively parallel proc. databases," Comp. Standards \& Interfaces, vol. 54, pp. 86 - 93, 2017.

[30] C. Gröger, "Building an industry 4.0 analytics platform," DatenbankSpektrum, vol. 18, no. 1, pp. 5-14, 2018.

[31] M. Gupta et al., "An attribute-based access control model for secure big data proc. in hadoop ecosystem," in ABAC. ACM, 2018, pp. 13-24.

[32] R. Hai et al., "Constance: An intelligent data lake system," in ICMD. ACM, 2016, pp. 2097-2100.

[33] — " "Query rewriting for heterogeneous data lakes," in ADBIS Springer, 2018, pp. 35-49.

[34] A. Y. Halevy et al., "Managing google's data lake: an overview of the goods system," IEEE Data Eng. Bull., vol. 39, no. 3, pp. 5-14, 2016.

[35] J. Herman et al., "Using big data for insights into sustainable energy consumption in industrial and mining sectors," Journal of Cleaner Production, vol. 197, pp. 1352 - 1364, 2018.

[36] J. Hui et al., "Integration of big data: A survey," in Data Science. Springer, 2018, pp. 101-121.

[37] M. Jarke, "Data spaces: Combining goal-driven and data-driven approaches in community decision and negotiation support," in GDN. Springer, 2017, pp. 3-14.

[38] M. Jarke et al., On Warehouses, Lakes, and Spaces. Springer, 2017, ch. 16, pp. 231-245.

[39] P. Jovanovic et al., A Unified View of Data-Intensive Flows in Business Intelligence Systems: A Survey. Berlin, Heidelberg: Springer Berlin Heidelberg, 2016, ch. 3, pp. 66-107.

[40] M. Karpathiotakis et al., "No data left behind: Real-time insights from a complex data ecosystem," in SoCC. ACM, 2017, pp. 108-120.

[41] N. Kasrin et al., "Semantic data management for experimental manufacturing tech." Datenbank-Spektrum, vol. 18, no. 1, pp. 27-37, 2018.

[42] L. Kassner et al., "The stuttgart it architecture for manufacturing," in ICEIS. Springer, 2017, pp. 53-80.

[43] P. Kathiravelu et al., "A dynamic dw platform for creating and accessing biomedical data lakes," in DMAH. Springer, 2017, pp. 101-120.

[44] P. P. Khine et al., "Data lake: a new ideology in big data era," ITM Web Conf., vol. 17, p. 11, 2018.

[45] H. Kondylakis et al., "Implementing a data management infrastructure for big healthcare data," in BHI. IEEE, 2018, pp. 361-364.

[46] J. R. Landis et al., "The measurement of observer agreement for categorical data," biometrics, pp. 159-174, 1977.

[47] T.-H.-Y. Le et al., "Big data driven architecture for medical knowledge management systems in intracranial hemorrhage diagnosis," in IUKM. Springer, 2018, pp. 214-225.

[48] $\mathrm{C}$. Li et al., "The design and application of astronomy data lake in china-vo," in ADASS, vol. 512. ASP, 2017, p. 157.

[49] A. Maccioni et al., "Crossing the finish line faster when paddling the data lake with kayak," $V L D B$, vol. 10, no. 12, pp. 1853-1856, 2017.

[50] C. Madera et al., "The next information architecture evolution: The data lake wave," in MEDES. ACM, 2016, pp. 174-180.

[51] K. P. Maksymowicz et al., "A holistic approach to testing biomedical hypotheses and analysis of biomedical data," in BDAS. Springer, 2016, pp. $449-462$.

[52] M. A. Martínez-Prieto et al., "Integrating flight-related information into a (big) data lake," in DASC. IEEE/AIAA, 2017, pp. 1-10.

[53] S. McCarthy et al., "Combining web and enterprise data for lightweight data mart construction," in DEXA. Springer, 2018, pp. 138-146.

[54] M. L. McHugh, "Interrater reliability: the kappa statistic," Biochemia medica, vol. 22, no. 3, pp. 276-282, 2012.

[55] J. McPadden et al., "A scalable data science platform for healthcare and precision medicine research," CoRR, vol. abs/1808.04849, 2018.

[56] S. D. Meena et al., "Data lakes - a new repository for big data analytics," Int. Journal of Adv. Research in CS, vol. 7, no. 5, pp. 65-67, 2016.

[57] N. Miloslavskaya et al., "Big data, fast data and data lake concepts," Procedia Computer Science, vol. 88, pp. 300 - 305, 2016.

[58] S. Mitrovic, "Specifics of the integration of business intelligence and big data technologies in the processes of economic analysis," Business Informatics, vol. 42, no. 4, pp. 40-46, 2017.

[59] D. Moher et al., "Preferred reporting items for systematic review and meta-analysis protocols," Systematic reviews, vol. 4, no. 1, p. 1, 2015.
[60] B. M. Mrozek et al., "Soft and declarative fishing of information in big data lake," IEEE Transactions on Fuzzy Systems, vol. 26, no. 5, pp. $2732-2747,2018$

[61] A. A. Munshi et al., "Data lake lambda architecture for smart grids big data analytics," IEEE Access, vol. 6, pp. 40 463-40 471, 2018.

[62] I. D. Nogueira et al., "Modeling data lake metadata with a data vault," CoRR, vol. abs/1807.04035, 2018.

[63] D. E. O'Leary, "Embedding ai and crowdsourcing in the big data lake," Intelligent Systems, vol. 29, no. 5, pp. 70-73, 2014.

[64] E. Pena et al., "Framework to use modern big data software tools to improve operations at the paranal observatory," in Proc. SPIE, vol. 10704, 2018, pp. $10704-10704-11$.

[65] A. Pomp et al., "Enabling semantics in enterprises," in ICEIS. Springer, 2018, pp. 428-450.

[66] C. Quix et al., "GEMMS: A generic and extensible metadata management system for data lakes," in CAiSE, 2016, pp. 129-136.

[67] K. Rajesh et al., "An introduction to data lake," i-manager's Journal on Information Technology, vol. 5, no. 2, pp. 1-4, 2016.

[68] R. Ramakrishnan et al., "Azure data lake store: A hyperscale distributed file service for big data analytics," in ICMD. ACM, 2017, pp. 51-63.

[69] B. Ramesh, Big Data Architecture. New Delhi: Springer India, 2015, ch. 2, pp. 29-59.

[70] S. Rangarajan et al., "Scalable architecture for personalized healthcare service rec. using big data lake," in ASSRI. Springer, 2018, pp. 65-79.

[71] P. Revathy et al., "Analysis of big data security practices," in iCATccT, 2017, pp. 264-267.

[72] D. L. Sackett, Evidence-based Medicine How to practice and teach EBM. WB Saunders Company, 1997.

[73] H. B. Sankaranarayanan et al., "Passenger reviews reference architecture using big data lakes," in Confluence, 2017, pp. 204-209.

[74] R. S. Santos et al., "Big data analytics in a public general hospital," in MOD. Springer, 2016, pp. 433-441.

[75] S. Sharma, "Expanded cloud plumes hiding big data ecosystem," Future Generation Computer Systems, vol. 59, pp. 63 - 92, 2016.

[76] G. Shlyuger, "Apply analytical grid processing to sensor data collections," in SPIE, vol. 10185, 2017, pp. 10185 - 10185 - 13.

[77] K. Singh et al., "Visual bayesian fusion to navigate a data lake," in FUSION, 2016, pp. 987-994.

[78] T. J. Skluzacek et al., "Klimatic: A virtual data lake for harvesting and distribution of geospatial data," in PDSW-DISCS, 2016, pp. 31-36.

[79] G. V. Solar et al., "Big data management: What to keep from the past to face future challenges?" DS and Eng., vol. 2, no. 4, pp. 328-345, 2017.

[80] R. K. Sreekala PK, "Data lake in the big data era: An overview," Library Herald, vol. 56, no. 1, pp. 11-15, 2018.

[81] M. K. Srinivasan et al., "State-of-the-art big data security taxonomies," in ISEC. ACM, 2018, pp. 16:1-16:7.

[82] J. Stefanowski et al., "Exploring complex and big data," Int. J. Appl. Math. Comput. Sci., vol. 27, no. 4, pp. 669-679, 2017.

[83] I. Suriarachchi et al., "Crossing analytics systems: A case for integrated provenance in data lakes," in e-Science. IEEE, 2016, pp. 349-354.

[84] — , "Provenance as essential infrastructure for data lakes," in IPAW, 2016, pp. 178-182.

[85] Y. Taher et al., "A service-based system for sentiment analysis and vis. of twitter data in realtime," in ICSOC. Springer, 2017, pp. 199-202.

[86] - "A context-aware analytics for processing tweets and analysing sentiment in realtime," in OTM. Springer, 2016, pp. 910-917.

[87] I. G. Terrizzano et al., "Data wrangling: The challenging journey from the wild to the lake," in CIDR, 2015, pp. 1-9.

[88] S. Tovernić et al., "Solution for detecting sensitive data inside a data lake," in MIPRO, 2018, pp. 1284-1288.

[89] M. University, "Systematic reviews: Using pico or pico," https://goo.gl/ fqPoCY 2018, accessed: 2018-12-20.

[90] W. Villegas-Ch et al., "Big data, the next step in the evolution of educational data analysis," in ICITS 2018. Springer, 2018, pp. 138-147.

[91] C. Walker et al., "Personal data lake with data gravity pull," in BDCloud. IEEE, 2015, pp. 160-167.

[92] R. Wenning et al., Compliance Using Metadata. Springer, 2018, ch. 3 , pp. 31-45.

[93] M. Wibowo et al., "Machine learning in data lake for combining data silos," in DMBD. Springer, 2017, pp. 294-306.

[94] S. Yadav et al., "Business data fusion," in Fusion, 2015, pp. 1876-1885.

[95] T. Yamada et al., "Interactive service for visualizing data assoc. using a self-org. structure of schemas," in SOCA. IEEE, 2017, pp. 230-233. 